Tucson. Instead, it underscores that seismic gaps can rupture in all sorts of ways, from lots of smaller quakes to just a few big ones.

Chile is an ideal laboratory in which to study such questions because it lies on the margin of a subduction zone, where the Nazca tectonic plate dives - or subducts - beneath the South American plate. Geological stress builds up and then is released in the occasional massive jolt. Chile is home to the largest earthquake ever recorded — one of magnitude 9.5 in 1960 - and accounts for more than one-quarter of the planet's total seismic-energy release.

Pisagua had not seen a major earthquake since 1877, when a tremor of around magnitude 9 ripped through the area. Seismic activity began to pick up last August, when a swarm of small earthquakes struck the area. Another set followed over the new year, and a third cluster occurred in March. These three swarms seem to have prepared the subduction zone to rupture in the big 1 April quake, says Onno Oncken, a geophysicist at the German Research Centre for Geosciences in Potsdam.

Until recently, researchers had thought that the next large earthquake in northern Chile would break the entire interface between the Nazca and South American plates, says Jean-Pierre Vilotte, a seismologist at the Paris Institute of Earth Physics. The Pisagua quake and a magnitude-7.6 aftershock two days later, "are a clear counterexample of this simplistic classification", he says. Together they ruptured just a small portion of the entire region at risk.

Intriguingly, the part of the subduction zone that broke was not the part that had built up the most stress, according to a ground-motion study of northern Chile's seismic gap by Marianne Métois, a geophysicist at the National Institute of Geophysics and Volcanology in Rome, and her colleagues (M. Métois et al. Geophys. J. Int. 194, 1283-1294; 2013). For some reason, the Pisagua quake released stress in areas that were not the most wound up. "A lot of energy remains to be released in north Chile," Métois says.

When the next one comes, seismologists plan to be ready. IPOC has added instruments to capture aftershocks from the 1 April quake and whatever might happen next. Dozens of new seismometers and global-positioning stations have been deployed by teams from Chile, Germany and France, says Sergio Barrientos, director of the National Seismological Centre at the University of Chile in Santiago.

For Sobiesiak, every little bit of data helps. Before the 1 April quake, she thought that the northern Chile seismic gap would rupture either to the north or to the south of the Pisagua area, but not right through it. Now she has some fresh thinking to do. "Each of these efforts really does bring us a step forward," she says.

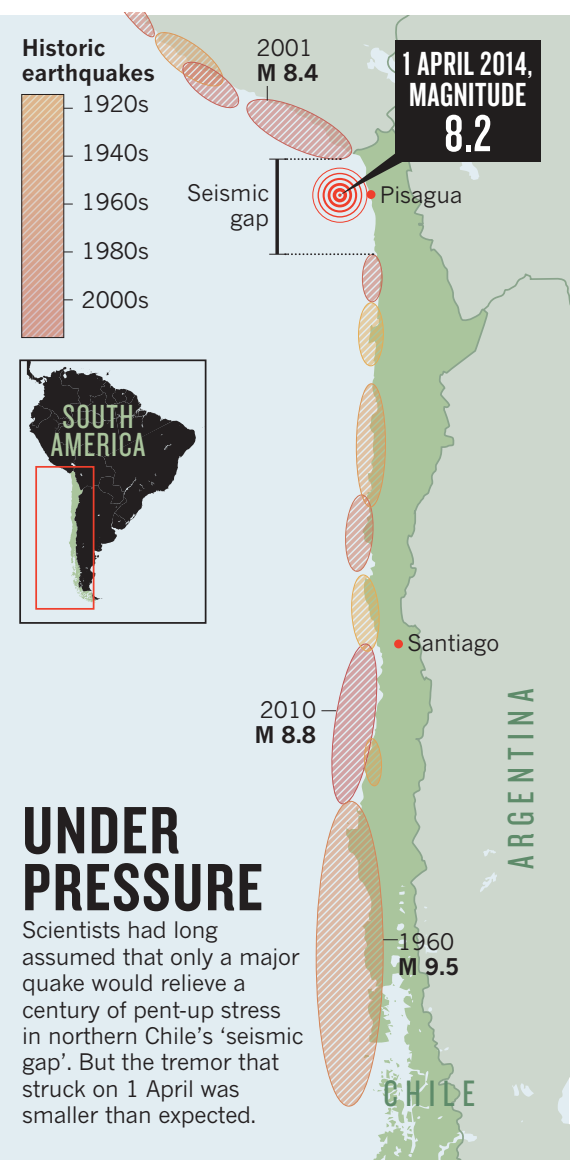

\title{
Quantum communications leap out of the lab
}

\author{
China begins work on super-secure network as 'real-world' trial successfully sends \\ quantum keys and data.
}

\section{BY JANE QIU}

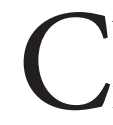
ybersecurity is a step closer to the dream of sending data securely over long distances using quantum physics - spurred by two developments.

This week, China will start installing the world's longest quantum-communications network, which includes a 2,000-kilometre link between Beijing and Shanghai. And a study jointly announced this week by the companies Toshiba, BT and ADVA, with the UK National Physical Laboratory in Teddington, reports "encouraging" results from a network field trial, suggesting that quantum communications could be feasible on existing fibre-optic infrastructure.
Conventional data-encryption systems rely on the exchange of a secret 'key' - in binary 0s and 1s - to encrypt and decrypt information. But the security of such a communication channel can be undermined if a hacker 'eavesdrops' on this key during transmission. Quantum communications use a technology called quantum key distribution (QKD), which harnesses the subatomic properties of photons to "remove this weakest link of the current system", says Grégoire Ribordy, co-founder and chief executive of ID Quantique, a quantum-cryptography company in Geneva, Switzerland.

The method allows a user to send a pulse of photons that are placed in specific quantum states that characterize the cryptographic key. If anyone tries to intercept the key, the act of eavesdropping intrinsically alters its quantum state - alerting users to a security breach. Both the US\$100-million Chinese initiative and the system tested in the latest study use QKD.

The Chinese network "will not only provide the highest level of protection for government and financial data, but provide a test bed for quantum theories and new technologies", says Jian-Wei Pan, a quantum physicist at the University of Science and Technology of China in Hefei, who is leading the Chinese project.

Pan hopes to test such ideas using the network, along with a quantum satellite that his team plans to launch next year (see Nature 492, $22-25 ; 2012)$. Together, he says, the technologies could perform further tests of fundamental 


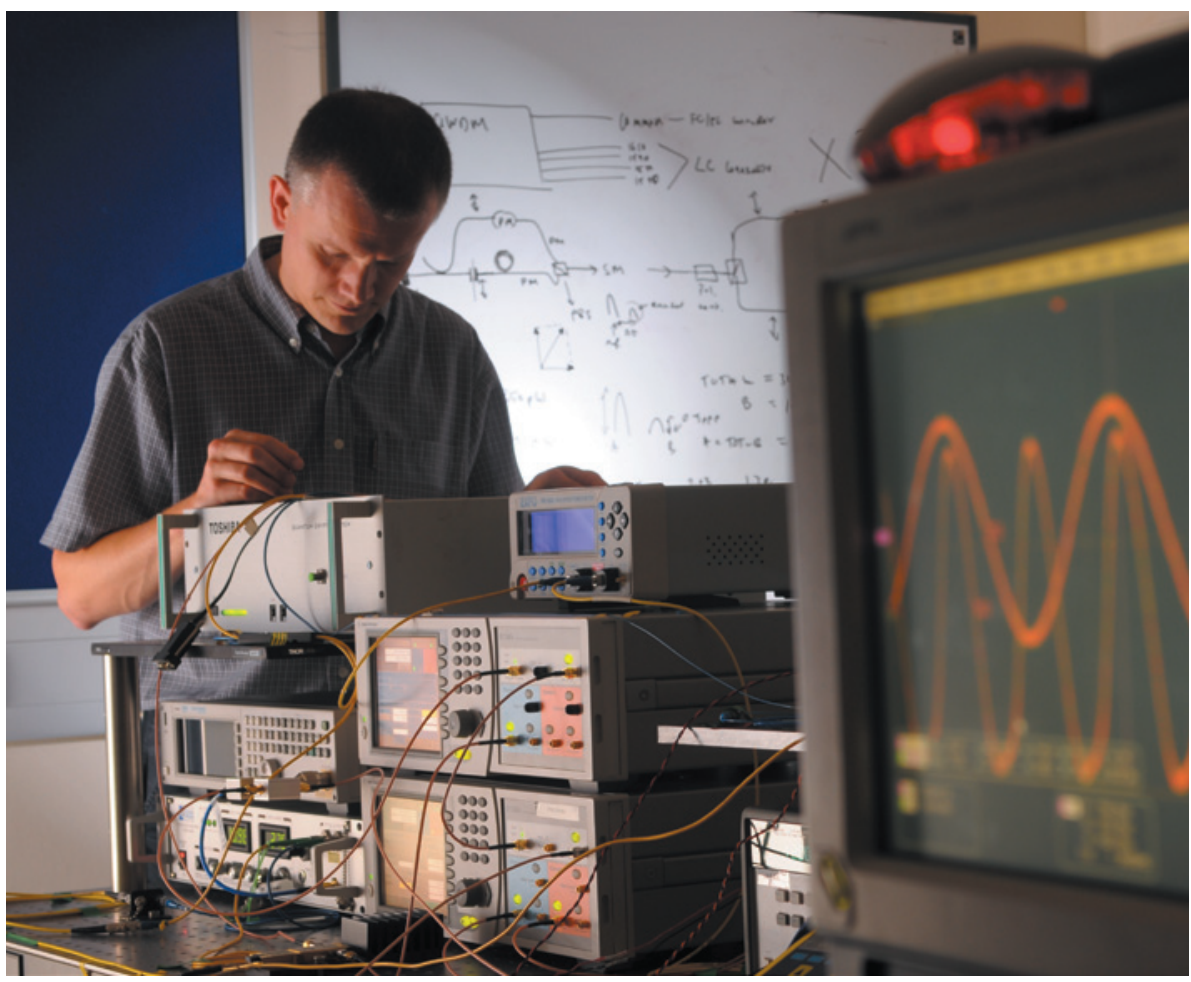

Engineer Andrew Sharpe works on Toshiba's quantum key distribution system in Cambridge, UK.

quantum theories over large scales (around 2,000 kilometres), such as quantum nonlocality, in which changing the quantum state of one particle can influence the state of another even if they are far apart, says Pan.

Sending single photons over long distances is one of the greatest problems in QKD because they tend to get absorbed by optical fibres, making the keys tricky to detect on the receiver's end.

This is "a big challenge for conventional detectors", says Hoi-Kwong Lo, a quantum physicist at the University of Toronto in Canada. But technological breakthroughs in recent years have significantly reduced the noise level of detectors while increasing their efficiency in detecting photons from just $15 \%$ to $50 \%$.

Vast improvements have also been made in the rate at which detectors can 'count' photon pulses - crucial in determining the rate at which quantum keys can be sent, and thus the speed of the network. Counting rates have been raised 1,000-fold, to about 2 gigahertz, says Lo.

The breakthroughs are pushing the distance over which quantum signals can be sent. Trials using 'dark fibres' - optical fibres laid down by telecommunications companies but lying unused - have sent quantum signals up to 100 kilometres, says Don Hayford, a researcher at Battelle, a technology-development company headquartered in Columbus, Ohio.

To go farther than that, quantum signals must be relayed at 'node points' - the quantum networks between Beijing and Shanghai, for instance, will require 32 nodes. To transmit photons over longer distances without the use of nodes would require a satellite.

China is not alone in its quantum-communication efforts. A team led by Hayford, together with ID Quantique, has started installing a 650-kilometre link between Battelle's headquarters and its offices in Washington DC. The partnership is also planning a network linking major US cities, which could exceed 10,000 kilometres, says Hayford, although it has yet to secure funding for that.

The Chinese and US networks will both use dark fibres to send quantum keys. But these fibres "are not always available and can be prohibitively expensive", says Andrew Shields, a quantum physicist at Toshiba Research Europe in Cambridge, UK. One way to sidestep the problem is to piggyback the photon streams onto the 'lit' fibres that transmit conventional telecommunications data. However, those conventional data streams are usually about a million times stronger than quantum streams, so the quantum data tend to be drowned out.

In the results announced this week, Shields and his colleagues were successful in achieving the stable and secure transmission of QKDs along a live lit fibre between two stations of the UK telecommunications company BT, 26 kilometres apart. The quantum keys were sent over several weeks at a high rate alongside four channels of strong conventional data on the same fibre.

The research builds on previous work in which Shields and his team developed a technique to detect quantum signals sent alongside noisy data in a 90-kilometre fibre, but in controlled laboratory conditions (K. A. Patel et al. Phys. Rev. X 2, 041010; 2012).

"Implementing QKD in the 'real world' is much more challenging than in the controlled environment of the lab, due to environmental fluctuations and greater loss in the fibre," says Shields.

The quantum keys in the latest study were sent alongside conventional data travelling at

\section{The breakthroughs are pushing the} distance over which quantum signals can be sent. 40 gigabits per second. "As far as I am aware, this is the highest bandwidth of data that has been multiplexed with QKD to date," add Shields.

He calculates that it would be possible to send QKD signals alongside 40 conventional data channels. Optical fibres usually carry between 40 and 160 telecommunications channels, meaning that quantum communication could be carried out with existing infrastructure.

"I find it an impressive piece of work that demonstrates the multiplexing of strong classical signals with quantum signals in the same fibre for the first time" in a field trial, says Lo. Removing the need for dark fibres, he says, is an important step in showing that QKD has the potential to be used in "real life".

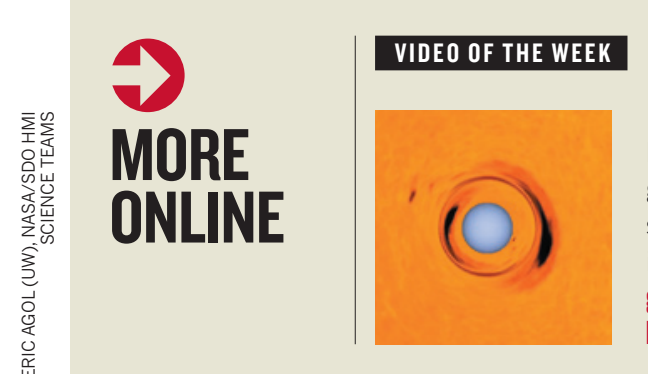

Planet hunter sees gravitational self-lensing in double star go.nature.com/ $13 \mathrm{mlxy}$

\section{MORE NEWS}

- An Earth-like planet in the habitable zone go.nature.com/tcxyzc

- Insect's 'female penis' extracts nourishment with semen go.nature.com/ cjuckd

- Archaic human epigenomes get mapped go.nature.com/hnzsye

\section{NATURE PODCAST}

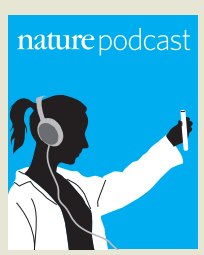

The worth of the Y chromosome; an unusual amateur scientist; and how to help corals breed nature.com/ nature/podcast 